\title{
Downregulation of FeSOD-A expression in Leishmania infantum alters trivalent antimony and miltefosine susceptibility
}

\author{
Ana Maria Murta Santi ${ }^{\dagger}$, Paula Alves Silva ${ }^{\dagger}$, Isabella Fernandes Martins Santos and \\ Silvane Maria Fonseca Murta* (0)
}

\begin{abstract}
Background: Superoxide dismutase (SOD), a central component of the antioxidant defence system of most organisms, removes excess superoxide anions by converting them to oxygen and hydrogen peroxide. As iron (Fe) SOD is absent in the human host, this enzyme is a promising molecular target for drug development against trypanosomatids.

Results: We obtained Leishmania infantum mutant clones with lower FeSOD-A expression and investigated their phenotypes. Our attempts to delete this enzyme-coding gene using three different methodologies (conventional allelic replacement or two different CRISPR/methods) failed, as FeSOD-A gene copies were probably retained by aneuploidy or gene amplification. Promastigote forms of WT and mutant parasites were used in quantitative reversetranscription polymerase chain reaction (RT-qPCR) and western blot analyses, and these parasite forms were also used to assess drug susceptibility. RT-qPCR and western blot analyses revealed that FeSOD-A transcript and protein levels were lower in FeSOD-A $A^{-/-1+}$ L. infantum mutant clones than in the wild-type (WT) parasite. The decrease in FeSOD-A expression in L. infantum did not interfere with the parasite growth or susceptibility to amphotericin B. Surprisingly, FeSOD-A $A^{-1-1+}$ L. infantum mutant clones were 1.5- to 2.0-fold more resistant to trivalent antimony and 2.4- to 2.7-fold more resistant to miltefosine. To investigate whether the decrease in FeSOD-A expression was compensated by other enzymes, the transcript levels of five FeSODs and six enzymes from the antioxidant defence system were assessed by RT-qPCR. The transcript level of the enzyme ascorbate peroxidase increased in both the FeSOD-A $A^{-/ /+}$mutants tested. The FeSOD- $A^{-/ /+}$mutant parasites were 1.4- to 1.75-fold less tolerant to oxidative stress generated by menadione. Infection analysis using THP-1 macrophages showed that $72 \mathrm{~h}$ post-infection, the number of infected macrophages and their intracellular multiplication rate were lower in the FeSOD-A $A^{-/ /+}$mutant clones than in the WT parasite.
\end{abstract}

Conclusions: The unsuccessful attempts to delete FeSOD-A suggest that this gene is essential in L. infantum. This enzyme plays an important role in the defence against oxidative stress and infectivity in THP-1 macrophages. FeSODA-deficient $L$. infantum parasites deregulate their metabolic pathways related to antimony and miltefosine resistance.

Keywords: Leishmania infantum, Iron superoxide dismutase, Knockout, Oxidative stress, Drug resistance

*Correspondence: silvane.murta@fiocruz.br

${ }^{\dagger}$ Ana Maria Murta Santi and Paula Alves Silva contributed equally to this work

Grupo de Genômica Funcional de Parasitos (GFP), Instituto René Rachou, Fiocruz Minas, Avenida Augusto de Lima 1715, Belo Horizonte, MG CEP: 30190-002, Brazil

\section{Background}

Leishmaniases are zoonotic or anthroponotic infectious diseases caused by the protozoan parasites of the genus Leishmania. More than 21 species of Leishmania are known to infect humans and are transmitted by the bite of more than 90 species of female sandflies. original author(s) and the source, provide a link to the Creative Commons licence, and indicate if changes were made. The images or other third party material in this article are included in the article's Creative Commons licence, unless indicated otherwise in a credit line to the material. If material is not included in the article's Creative Commons licence and your intended use is not permitted by statutory regulation or exceeds the permitted use, you will need to obtain permission directly from the copyright holder. To view a copy of this licence, visit http://creativecommons.org/licenses/by/4.0/. The Creative Commons Public Domain Dedication waiver (http://creativeco mmons.org/publicdomain/zero/1.0/) applies to the data made available in this article, unless otherwise stated in a credit line to the data. 
Leishmaniasis mostly affects vulnerable populations and is detected in 98 countries across Europe, Africa, Asia, the Americas, and Oceania. More than 30,000 cases of visceral leishmaniasis and one million cases of cutaneous leishmaniasis are registered worldwide each year [1].

Only a few drugs are currently available for leishmaniasis treatment: pentavalent antimonials $\left(\mathrm{Sb}^{\mathrm{V}}\right)$, amphotericin $\mathrm{B}$ (AMB) and formulations, and miltefosine. The therapeutic failure of leishmaniasis is a major concern, and is probably related to the parasite's resistance to treatment and the patient's nutritional status, age, sex, and immunity. Another major concern with available therapies is their toxicity and side effects [2].

Given the present scenario, the development of new non-toxic and more effective drugs for the treatment of leishmaniasis is imperative. In this context, iron superoxide dismutase A (FeSOD-A) has garnered significant attention as a possible molecular target. It is an important enzyme in the antioxidant defence system that protects the parasites against superoxide radicals $\left(\mathrm{O}_{2}{ }^{-}\right)$, which are converted to oxygen $\left(\mathrm{O}_{2}\right)$ and hydrogen peroxide $\left(\mathrm{H}_{2} \mathrm{O}_{2}\right)$ [3]. $\mathrm{H}_{2} \mathrm{O}_{2}$ is then metabolised by different enzymes with peroxidase activity, such as tryparedoxin peroxidase (TXNPx), ascorbate peroxidase (APX), peroxiredoxins (PRXs), and glutathione peroxidases (GPXs) [4].

The metalloenzyme SOD (EC 1.15.1.1) is a key component in the antioxidant defence system of most organisms and exhibits different metal cofactors at its active site [5]. In trypanosomatids, SODs have iron (Fe) in their structure and are classified as FeSOD-A expressed in the mitochondria [6, 7], FeSOD-B1 and FeSOD-B2 located in the glycosome [8], and FeSOD-C detected in the mitochondria [6]. As FeSOD is absent in the human host, this enzyme can serve as a promising molecular target for drug development against trypanosomatids.

FeSOD-deficient $L$. tropica was shown to be more sensitive to oxidative stress and FeSOD-deficient $L$. donovani had decreased ability to infect murine macrophages [9]. In addition, L. amazonensis deficient in FeSOD-A was found to be more sensitive to oxidative stress and less effective in producing lesions in mice [10]. Another study showed that a $L$. donovani strain isolated from a patient was resistant to miltefosine and overexpressed FeSODA [11]. L. braziliensis and L. infantum overexpressing FeSOD-A were more resistant to trivalent antimony and more tolerant to oxidative stress than their respective sensitive counterparts [12].

Considering the importance of this enzyme for the parasites and the potential for using FeSOD-A as a molecular target, here we investigate the impact of FeSOD-A downregulation in L. infantum on the drug susceptibility phenotype. We also evaluate tolerance to oxidative stress and infectivity by THP-1 macrophages, and determine the transcript levels of 11 other enzymes involved in the antioxidant defence system.

\section{Methods}

L. (Leishmania) infantum RPV (MHOM/BR/2002/ LPC-RPV) promastigotes were grown at $26{ }^{\circ} \mathrm{C}$ in M199 medium (Gibco) supplemented with $40 \mathrm{mM}$ HEPES ( $\mathrm{pH}$ 7.4), $5 \mu \mathrm{g} / \mathrm{mL}$ hemin, $2 \mu \mathrm{g} / \mathrm{mL}$ biopterin, $1 \mu \mathrm{g} / \mathrm{mL}$ biotin, $2 \mathrm{mM}$ L-glutamine, $500 \mathrm{U} / \mathrm{mL}$ penicillin, $50 \mu \mathrm{g} / \mathrm{mL}$ streptomycin, and $10 \%$ inactivated fetal bovine serum [13]. Cultures were maintained by performing two weekly passages in which $1 \times 10^{6}$ parasites were inoculated in $5 \mathrm{~mL}$ of medium. All experiments were performed using promastigotes in the logarithmic growth phase unless otherwise stated.

The FeSOD-A (LINF_080007900) knockout attempt was initially performed by two rounds of gene replacement by homologous recombination using neomycin phosphotransferase (NEO) and hygromycin phosphotransferase (HYG) as selectable markers [14]. The homology arms used for homologous recombination flank the FeSOD-A coding sequence and are 518 bp long in the $5^{\prime}$ UTR and 459 bp long in the $3^{\prime} U T R$. Other knockout attempts were made using the CRISPR/Cas9 system.

The first attempt to knock out FeSOD-A using CRISPR was performed according to Zhang et al. [15]. The singleguide RNAs (sgRNAs) were selected using the Eukaryotic Pathogen CRISPR gRNA Design Tool (http://gRNA. ctegd.uga.edu), and the protospacer sequences were ligated into the pSPneoHHsgRNAaH vector that had been previously digested with the restriction enzyme BbsI (New England Biolabs). Parasites containing the plasmid pLPhygCas9 were independently transfected with two different versions of the plasmid pSPneoHHsgRNAaH, containing sgRNAs 144 or 282 , and a donor DNA to insert stop codons within the FeSOD-A coding sequence.

A second attempt to generate $F e S O D-A$ null mutants using CRISPR/Cas9 was performed as previously described by Beneke et al. [16]. The plasmid pTB007, which has hygromycin as a resistance marker, was used to express SpCas9 and T7RNAP. Parasites carrying this plasmid and successfully expressing Cas9 were transfected with donor DNAs and templates for guide RNAs chosen with the LeishGEdit tool. Plasmids pTNeo v1 and pTBlast v1 were used for PCR amplification of the donors with 30 bp-long homology arms. sgRNA templates were generated by PCR using the G00 primer. All primers used to construct the plasmids and DNA fragments are listed in Additional file 1: Table S1.

All transfections were performed as previously described [14]. The selection of Leishmania clones was done by plating the parasites in semi-solid M199 
medium, and selective drugs were added as per the resistance markers carried by the mutants as follows: $40 \mu \mathrm{g} / \mathrm{mL}$ G418 (Gibco), $400 \mu \mathrm{g} / \mathrm{mL}$ hygromycin B (Invitrogen), or $10 \mu \mathrm{g} / \mathrm{mL}$ blasticidin (Gibco). After that, selection drugs were used in the weekly passages of the cultures, but all experiments to assess the parasites' phenotype were conducted in the absence of selection drugs.

Deletion assessment was performed by PCR using primers to amplify the $F e S O D-A$ coding sequence and primers to evaluate the replacement of $F e S O D-A$ alleles by resistance marker sequences (Additional file 1: Table S1). Protein levels were assessed by western blotting using the polyclonal antibody anti-FeSOD-A [17] at a dilution of 1:500. Densitometric analysis was performed to compare FeSOD-A levels with the $\alpha$-tubulin reference.

To evaluate the growth of the parasite, $1 \times 10^{6}$ promastigotes $/ \mathrm{mL}$ were inoculated in M199 medium, and the parasite concentration was determined daily using the $\mathrm{Z} 1$ Coulter Counter (Beckman Coulter) cell counter.

To evaluate the parasite's susceptibility to antimony, AMB, miltefosine, and menadione, $2 \times 10^{6}$ promastigotes were incubated in $1 \mathrm{~mL}$ M199 medium containing various concentrations of drugs. The number of parasites grown in the absence and presence of the drug after $48 \mathrm{~h}$ of incubation was determined using the Z1 Coulter Counter (Beckman Coulter). The 50\% growth inhibitory concentration $\left(\mathrm{IC}_{50}\right)$ was determined using the non-linear regression-variable slope model as per the equation "log (inhibitor) vs. response" in GraphPad Prism v.8.2.0.

To assess transcript levels, quantitative reverse-transcription PCR (RT-qPCR) analysis was performed using the cDNA of wild-type (WT) parasites and mutants. Promastigotes (approximately $10^{8}$ cells) were harvested and resuspended in $1 \mathrm{~mL}$ TRIzol Reagent (Invitrogen), and total RNA was extracted using the chloroform method. The RNA was treated with DNase I (Ambion), and the cDNA was obtained using Superscript II reverse transcriptase (Invitrogen) according to the manufacturer's instructions. All cDNA samples were diluted to $100 \mathrm{ng} /$ $\mu \mathrm{L}$ and used in the RT-qPCR amplification reaction performed using 1X SYBR GREEN master mix (Applied Biosystems) and the specific primers listed in Additional file 1: Table S1. Specific primers for each enzyme were designed using conserved nucleotide regions to amplify the different gene copies found in TritrypDB (tritrypdb. org). The levels of transcripts of the following enzymes were evaluated: six FeSODs (SODA [LINF_080007900], putative SODB1 [LINF_320024000], SODB2 [LINF_320024100], SOD putative [LINF_300033000], SOD putative [LINF_320033200], and SOD putative [LINF_340012900]) and six enzymes from the antioxidant defence system (APX [LINF_340005600], NADHdependent fumarate reductase [FRD; LINF_350013000,
LINF_350016600, and LINF_350016700], TXNPx [LINF_150018600, LINF_150018800, and LINF_150019000], type II GPX-like TXNPx [LINF_260013100], GPX putative [LINF_360038100], and PRX [LINF_230005400]). The DNA polymerase gene (LINF_160021500) was used as a constitutive normaliser. Amplifications were performed using a QuantStudio ${ }^{\mathrm{TM}}$ 12 K Flex system (Thermo Fisher Scientific). The PCR conditions were as follows: initial denaturation step at $95^{\circ} \mathrm{C}$ for $5 \mathrm{~min}$, followed by 40 cycles of denaturation at $95{ }^{\circ} \mathrm{C}$ for $15 \mathrm{~s}$, annealing at $60^{\circ} \mathrm{C}$ for $15 \mathrm{~s}$, and extension at $60{ }^{\circ} \mathrm{C}$ for $30 \mathrm{~s}$. Fluorescence was measured after each cycle. Transcript levels were determined using the comparative $C_{\mathrm{T}}$ method ( $2^{-\Delta \Delta \mathrm{CT}}$ method).

Cells derived from the human monocytic strain THP-1 were cultured in complete Rowell Park Memorial Institute (RPMI)-1640 medium (supplemented with 10\% fetal bovine serum, $2 \mathrm{mM}$ glutamine, $100 \mathrm{U} / \mathrm{mL}$ penicillin, and $100 \mu \mathrm{g} / \mathrm{mL}$ streptomycin). Monocytes were differentiated into macrophages by the addition of $20 \mathrm{ng} / \mathrm{mL}$ phorbol myristate acetate (PMA). After $72 \mathrm{~h}$, the macrophages were infected with Leishmania cultures on the second day of the stationary phase (10 parasites per macrophage) for $6 \mathrm{~h}$. The parasites that failed to infect the macrophages were washed away, and the infected macrophages were incubated for $72 \mathrm{~h}$ in RPMI-1640 medium. The infectivity of mutant clones was assessed immediately after the conclusion of the $6 \mathrm{~h}$ incubation period as well as after $72 \mathrm{~h}$. The slides were stained with rapid panoptic (Laborclin) and photographed, and the infection was quantified by counting intracellular amastigotes using ImageJ free software.

\section{Statistical analysis}

For all experiments, at least three technical replicates were performed for each of the three biological replicates. Data were analysed using GraphPad Prism v.8.2.0. Statistical significance was set at $p<0.05$. The $p$-values were reported as per the GraphPad Prism format, where ns $(p>0.05),{ }^{*}(p \leq 0.05),{ }^{* * *}(p \leq 0.01),{ }^{* * * *}(p \leq 0.001)$, and **:**: $(p \leq 0.0001)$.

\section{Results}

The first attempt to knock out FeSOD-A in L. infantum was performed using two rounds of gene replacement by homologous recombination. This attempt was unsuccessful, leading to the retention of one copy of the gene by aneuploidy or gene amplification even after the correct integration of two different selectable markers replacing FeSOD-A. The presence of both NEO and HYG cassettes and their correct integration into the $L$. infantum genome replacing $\mathrm{FeSOD}-\mathrm{A}$ was confirmed by PCR in all tested mutant clones (Fig. 1a, b). PCR was 


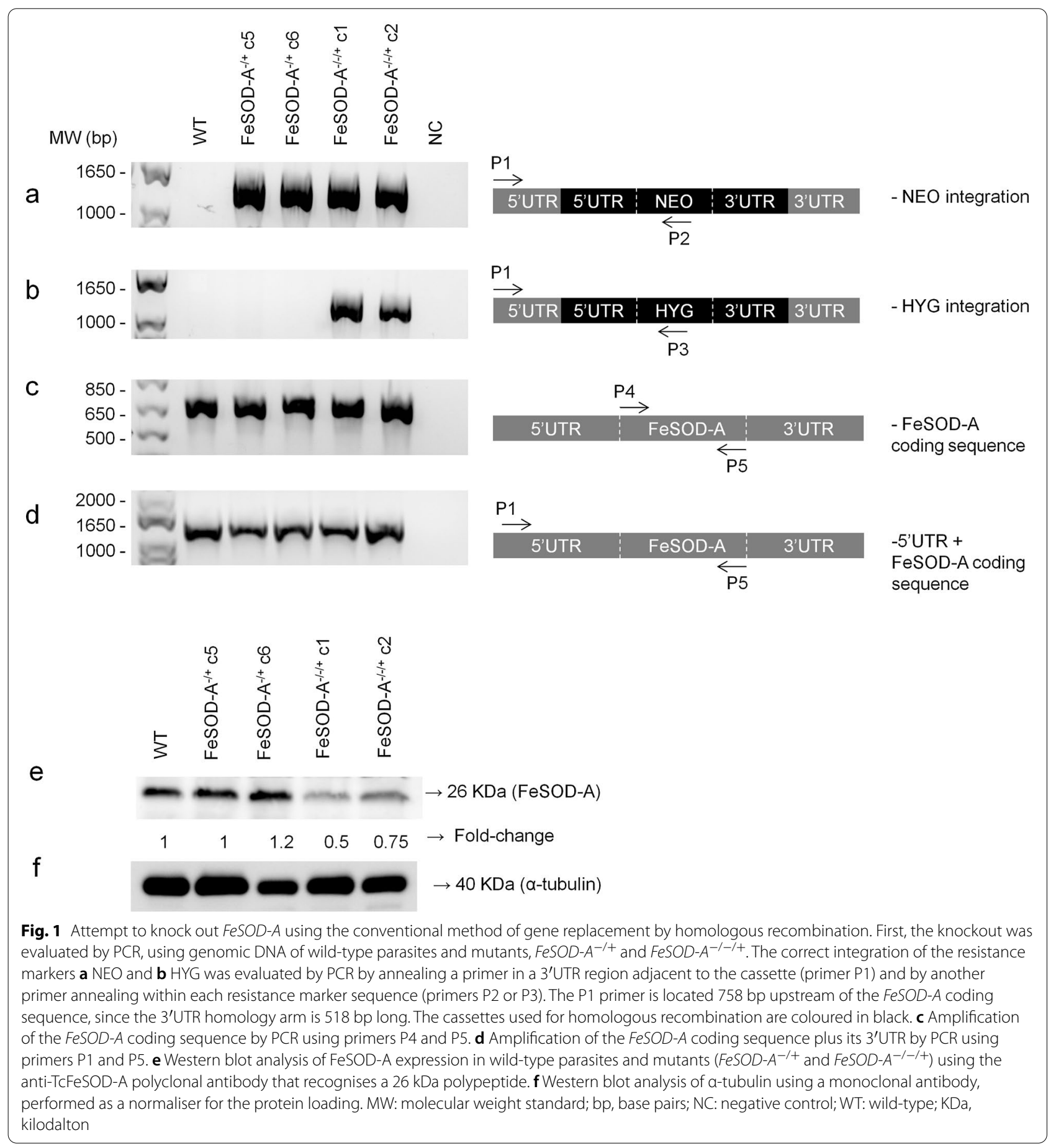

also used to verify the deletion of FeSOD-A in these parasites, and the results demonstrated its retention in the mutant parasites. The obtained clones were named FeSOD $-A^{-/-/+}$(Fig. 1c). Similarly, the attempts to knock out the FeSOD-A gene by CRISPR were unsuccessful. In all attempts, copies of the gene were retained by aneuploidy or gene amplification (Additional file 2: Figure S1 and Additional file 3: Figure S2).

FeSOD-A protein expression was evaluated in WT parasites, FeSOD- $A^{-1+}$ clones $\mathrm{C} 5$ and $\mathrm{C} 6$, and FeSOD$A^{-1-1+}$ clones $\mathrm{C} 1$ and $\mathrm{C} 2$ by western blotting using a polyclonal antibody produced against the recombinant 
FeSOD-A protein. This antibody recognised a $26 \mathrm{kDa}$ polypeptide in all the tested parasites. To normalise expression, FeSOD-A signals were compared to the signal of the $\alpha$-tubulin by densitometric analysis. The WT parasites and FeSOD-A $A^{-1+}$ clone $\mathrm{C} 5$ had the same levels of FeSOD-A protein, but FeSOD- $A^{-1+}$ clone 6 had a $20 \%$ increase in FeSOD-A expression when compared to the WT (Fig. 1e). On the other hand, the FeSOD$A^{-/-/+}$mutant parasites showed lower levels of FeSODA than the WT parasite, as evident from a $50 \%$ decrease in clone 1 and a $25 \%$ decrease in clone 2 .

The growth of promastigote forms of WT, FeSOD$A^{-/+}$, and FeSOD $-A^{-1-/+}$ mutant clones was followed by daily parasite counting. No growth differences were observed between WT and mutant parasites (Additional file 4: Figure S3).

We investigated whether lower FeSOD-A expression alters the susceptibility of parasites to leishmanicidal drugs such as trivalent antimony $\left(\mathrm{Sb}^{\mathrm{III}}\right)$, miltefosine, and AMB. FeSOD $-A^{-/-/+}$mutants were found to be more resistant to $\mathrm{Sb}^{\mathrm{III}}$ and miltefosine than the WT parasite. In the case of $\mathrm{Sb}^{\mathrm{III}}$, the WT parasite presented an $\mathrm{IC}_{50}$ value of $66.7 \mu \mathrm{M}$ and $\mathrm{FeSOD-A^{-/-/+ }}$ clones $\mathrm{C} 1$ and $\mathrm{C} 2$ had $\mathrm{IC}_{50}$ values of 130.7 and $97.5 \mu \mathrm{M}$, respectively, which were 2.0- and 1.5-fold higher than the WT $\mathrm{IC}_{50}$ (Fig. 2a). We also evaluated the susceptibility to miltefosine and found an $\mathrm{IC}_{50}$ value of $7.1 \mu \mathrm{M}$ for the WT parasite and 16.9 and $19.1 \mu \mathrm{M}$ for $\mathrm{FeSOD}-A^{-/-/+}$ clones $\mathrm{C} 1$ and $\mathrm{C} 2$, respectively, indicating 2.4- and 2.7 fold higher values than the WT $\mathrm{IC}_{50}$ (Fig. 2b). However, WT and FeSOD- $A^{-/-/+}$clones $\mathrm{C} 1$ and $\mathrm{C} 2$ showed the same susceptibility to $\mathrm{AMB}$, with their $\mathrm{IC}_{50}$ values ranging from 0.11 to $0.12 \mu \mathrm{M}$ (Fig. 2c).

The results of RT-qPCR analyses were in line with the western blot findings and showed that FeSOD$A$ (LINF_080007900) transcript levels were 56\% and $46 \%$ lower in FeSOD-A $A^{-1-+}$ L. infantum mutant clones $\mathrm{C} 1$ and $\mathrm{C} 2$, respectively, than in the WT parasite (Fig. 3). To investigate whether the decrease in FeSOD-A expression was compensated by an increase in some other enzymes, the transcript levels of five FeSODs (FeSOD-B1, FeSOD-B2, and three SOD putative LINF_300033000, LINF_320033200, and LINF_340012900) and six other enzymes from the antioxidant defence system (APX, TXNPx, PRX, GPX, FDR, and type II [GPX-like] TXNPx) were assessed by RT-qPCR. The data demonstrated an increase of $63 \%$ and $51 \%$ in APX transcript levels in L. infantum mutant clones $\mathrm{C} 1$ and $\mathrm{C} 2$ as compared with that in the WT parasite, respectively. In addition, FeSOD- $A^{-/-/+}$ clone $\mathrm{C} 1$ showed an increase in TXNPx and SOD putative (LINF_340012900) expression. FeSOD- $A^{-/-/+}$ clone $\mathrm{C} 2$ had an increase in FeSOD putative SODB1 and SODB2 and SOD putative (LINF_300033000) expression (Fig. 3).

To further evaluate the mutant phenotype, WT and FeSOD- $A^{-/-/+}$mutant L. infantum parasites were incubated with increasing concentrations of menadione to investigate the effect of allelic replacement of FeSOD-A on protection against oxidative stress. Figure $2 \mathrm{~d}$ shows the percentage of parasites grown at different concentrations of menadione after $48 \mathrm{~h}$. The mutant parasites were 1.4- and 1.75-fold less tolerant to menadione than the WT parasite. While the WT parasite presented an $\mathrm{IC}_{50}$ value of $1.4 \mu \mathrm{M}$, the FeSOD- $A^{-/-/+}$mutant clones $\mathrm{C} 1$ and $\mathrm{C} 2$ presented $\mathrm{IC}_{50}$ values of 1.0 and $0.8 \mu \mathrm{M}$, respectively.

To evaluate whether the decrease in FeSOD-A expression changes the fitness of intracellular amastigote forms, we performed experimental infection of human macrophages THP-1 with WT and FeSOD- $A^{-/ /}$mutant parasites. No difference in the infectivity of macrophages or intracellular multiplication rate was observed between the WT and FeSOD- $A^{-/-1}$ clones $\mathrm{C} 1$ and $\mathrm{C} 2$ mutant parasites $6 \mathrm{~h}$ after infection (Fig. 4). However, FeSOD$A^{-/-/+}$mutants showed a lower number of infected macrophages (Fig. 4a), and fewer intracellular amastigotes were observed $72 \mathrm{~h}$ after infection (Fig. 4b).

\section{Discussion}

In this study, we attempted to perform FeSOD-A knockout in L. infantum using three different methodologies (conventional allelic replacement and two CRISPR/ Cas9 methods), but the copies of the gene were always retained, probably by aneuploidy or gene amplification [18]. The inability to delete FeSOD-A using three different methods suggests the essential role of this gene in $L$. infantum. It was recently demonstrated that FeSOD-A could not be deleted in L. amazonensis [10].

Several studies have shown that FeSOD-A is related to $\mathrm{Sb}^{\mathrm{III}}$ and miltefosine susceptibility. Tessarollo et al. [12] reported higher activity of FeSOD enzymes in L. infantum and L. braziliensis resistant to $\mathrm{Sb}^{\mathrm{III}}$. The authors also observed that L. infantum and L. braziliensis become more resistant to $\mathrm{Sb}^{\mathrm{III}}$ following FeSOD-A overexpression [12]. Getachew and Gedamu [19] demonstrated that $L$. donovani overexpressing FeSOD-A was more resistant to miltefosine [19]. Another study reported that a miltefosine-resistant $L$. donovani isolate overexpressed FeSOD-A and demonstrated increased enzyme activity as compared to the susceptible isolate [11].

To our knowledge, this is the first study to assess the susceptibility of $\mathrm{FeSOD}-\mathrm{A}^{-1-1+}$ mutant parasites to leishmanicidal drugs. Surprisingly, the FeSOD- $A^{-/-/+}$ $L$. infantum mutant clones obtained in the present study were more resistant to both $\mathrm{Sb}^{\mathrm{III}}$ and miltefosine than the WT. What may seem like a paradoxical result 


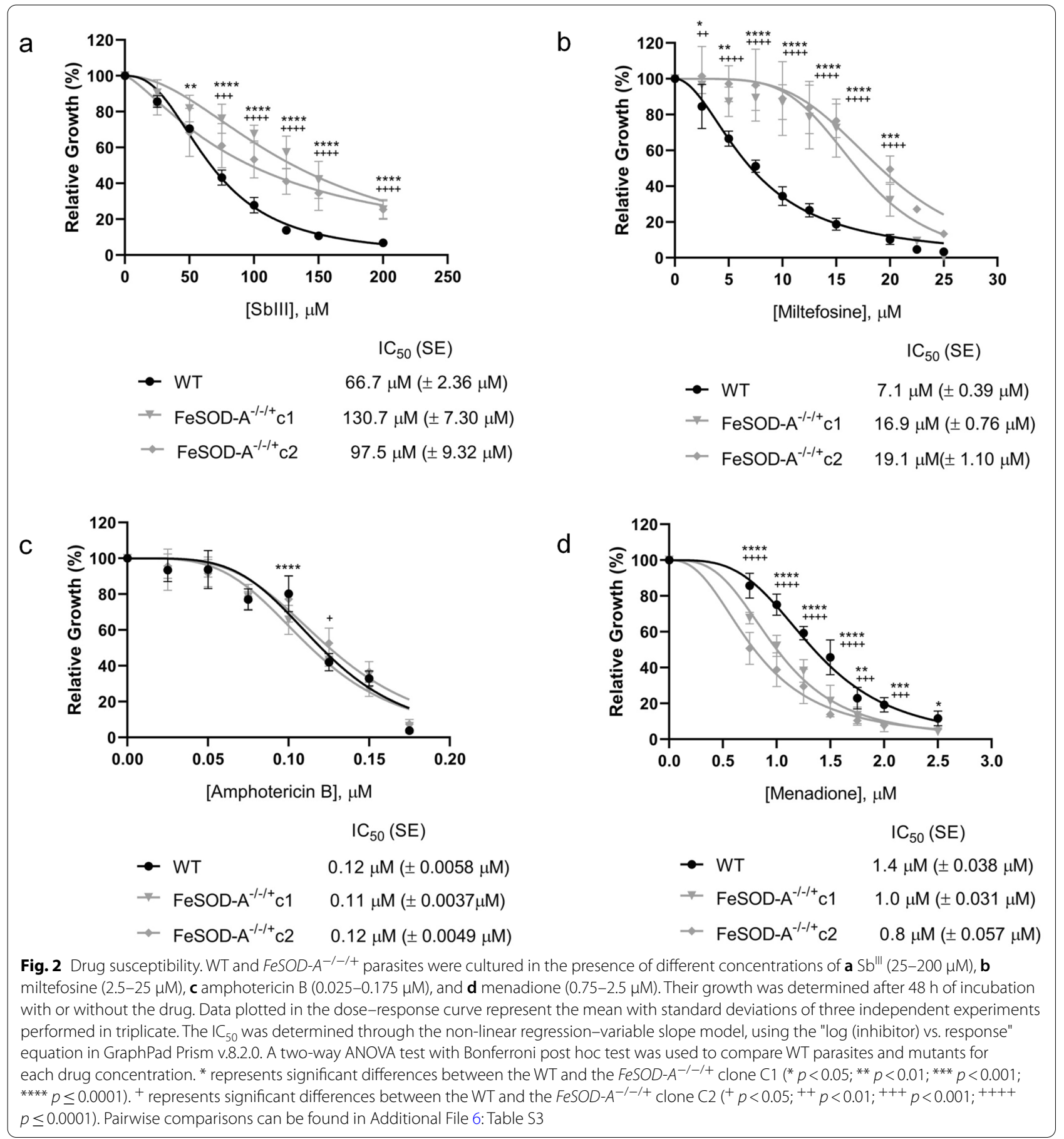

actually demonstrates the deregulation in the oxidative stress defence pathways and the ability of the parasite likely to compensate for the lower FeSOD-A expression. Interestingly, the existence of FeSOD-C in trypanosomatids was demonstrated in a study conducted by Dufernez et al. [6]. These authors showed that FeSOD-C, like FeSOD-A, is located in the mitochondria. MitoProt
II analysis indicated that the protein encoded by the LINF_300033000 gene is very likely to be exported to the mitochondria (Additional file 5: Table S2), suggesting that LINF_300033000 would be FeSOD-C in L. infantum. Thus, we initially hypothesised that this enzyme could compensate for the role of FeSOD-A. However, despite the presence of FeSOD-C in the mitochondria 


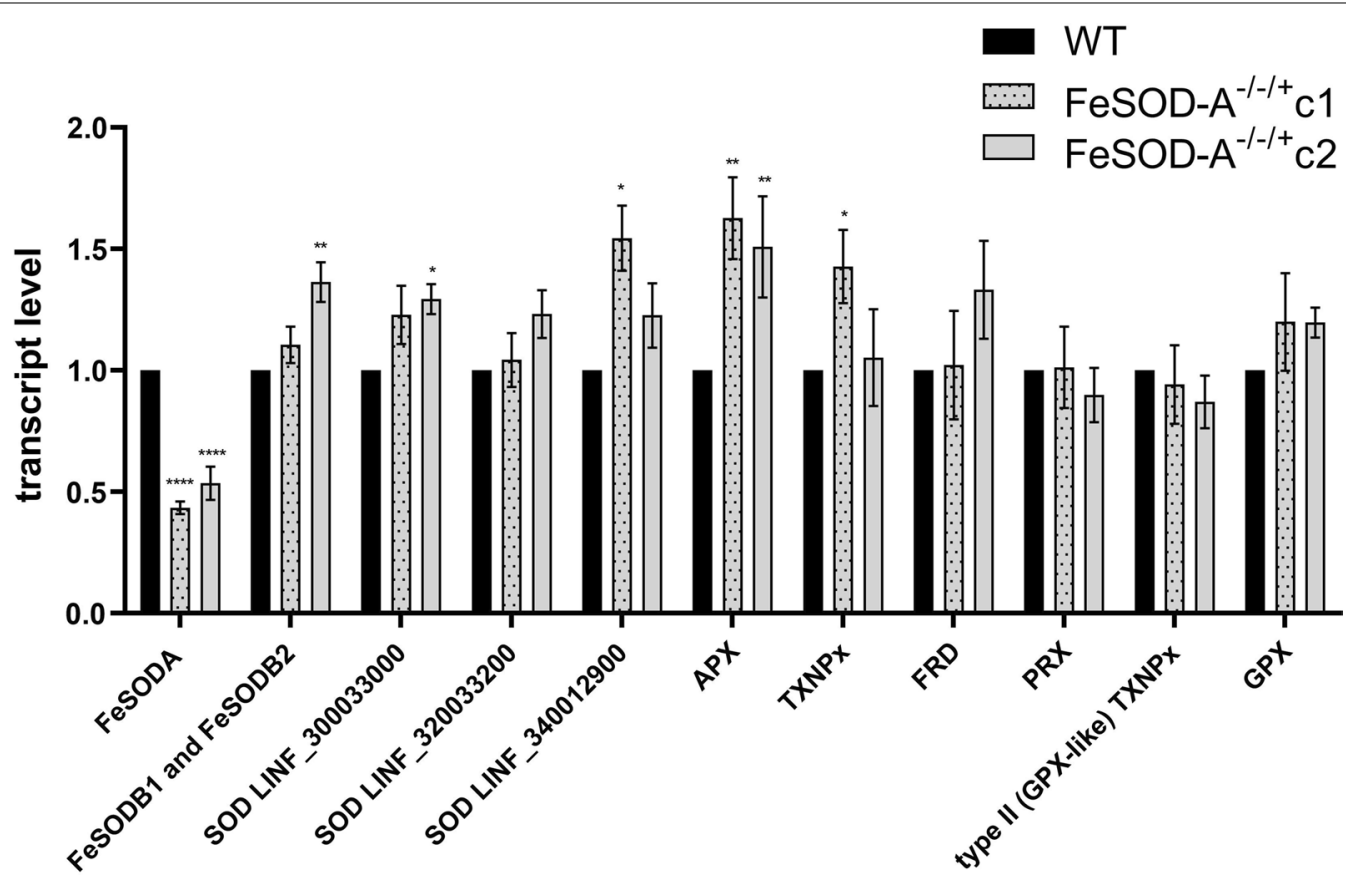

Fig. 3 Transcription levels of superoxides, peroxidases, and fumarate reductase were assessed by RT-qPCR in wild-type parasites and FeSOD-A ${ }^{-/-/+}$ mutants. DNA polymerase gene (LINF_160021500) was used as constitutive normaliser, and the fold change was calculated by the $2^{-\triangle \Delta C t}$ method. Enzymes analysed: iron superoxide dismutase SODA (LINF_080007900_FeSOD-A), iron superoxide dismutase - putative SODB1 (LINF_320024000—FeSOD-B1), iron superoxide dismutase - putative SODB2 (LINF_320024100—FeSOD-B2), superoxide dismutase—putative (SOD LINF_300033000), superoxide dismutase — putative (SOD LINF_320033200) and superoxide dismutase — putative (SOD LINF_340012900), ascorbate peroxidase (LINF_340005600—APX), tryparedoxin peroxidase (LINF_150018600, LINF_150018800, and LINF_150019000—TXNPX), NADH-dependent fumarate reductase (LINF_350013000, LINF_350016600, and LINF_350016700 FRD), peroxiredoxin (LINF_230005400_PRX), type II (glutathione peroxidase-like) tryparedoxin peroxidase (LINF_260013100 -type II (GPX-like) TXNPX), glutathione peroxidase-putative (LINF_360038100_GPX). Ordinary one-way ANOVA test with Bonferroni post hoc test was used to compare WT parasites and mutants for each gene separately. ${ }^{*}$ represents significant differences in relation to the wild-type parasite ${ }^{*} p<0.05 ;{ }^{* *} p<0.01$; ${ }^{* *} p<0.001$; $\left.{ }^{* * * *} p \leq 0.0001\right)$. Pairwise comparisons: FeSODA WT vs. C1 $F(2,19)=57.84, p<0.0001$; FeSODA WT vs. $C 2 F(2,19)=57.84, p<0.0001$; FeSODB1 and FeSODB2 WT vs. C2 F(2, $21)=8.596, p=0.0012 ;$ SOD LINF_300033000 WT vs. C2 $F(2,17)=5.314 ; p=0.0147 ;$ SOD LINF_340012900 WT vs. C1 $F(2,13)=4.993, p=0.0152 ; A P X$ WT vs. $C 1 F(2,11)=14.44, p=0.0013$; APXWT vs. $C 2 F(2,11)=14.44, p=0.0058 ;$ TXNPx WT vs. $C 1 F(2,13)=4.463, p=0.0241$

of the parasites, it was impossible to knock out FeSODA expression. Furthermore, a significant increase in FeSOD-C transcript levels was detected only in FeSOD$\mathrm{A}^{-/ /+}$clone $\mathrm{C} 2$, which also had an increase in FeSOD putative SODB1 and SODB2 expression. In contrast, FeSOD-A $^{-/-1+}$ clone $\mathrm{C} 1$ showed an increase in SOD putative (LINF_340012900) and TXNPx expression. The differing responses of the clones to the decrease in FeSOD-A expression suggest that the process of retention of the FeSOD-A gene copy by aneuploidy or gene amplification occurred in different ways between them. This heterogeneity is normal because Leishmania relies on aneuploidy as an adaptation mechanism [20]. Moreover, single-cell sequencing has confirmed the presence of different karyotypes within the same Leishmania clone $[21,22]$.

Among all evaluated enzymes, only APX transcript levels increased in both FeSOD-A $A^{-/ /+}$clones. This redox enzyme of the trypanothione pathway converts $\mathrm{H}_{2} \mathrm{O}_{2}$ into water molecules, regulating oxidative stress in Leishmania [23]. Thus, an increase in APX transcript levels may favour the resistance of $\mathrm{FeSOD}-\mathrm{A}^{-/-/+}$mutant clones to $\mathrm{Sb}^{\mathrm{III}}$ and miltefosine. Notably, L. braziliensis overexpressing APX was found to be eight times as resistant to $\mathrm{Sb}^{\mathrm{III}}$ and 1.8 times as resistant to $\mathrm{H}_{2} \mathrm{O}_{2}$ as the WT parasite [24].

Our results show that FeSOD-A downregulation in $L$. infantum did not interfere with the pathogen's susceptibility to AMB. Some factors can contribute to resistance to AMB, such as the lower drug binding to the membrane, owing to the changes in the sterol profile and membrane fluidity, AMB efflux, and free radical scavenger activity $[25,26]$. However, downregulation of only one enzyme of the antioxidant defence system may not have a greater impact on resistance to this drug, as demonstrated in our results. 

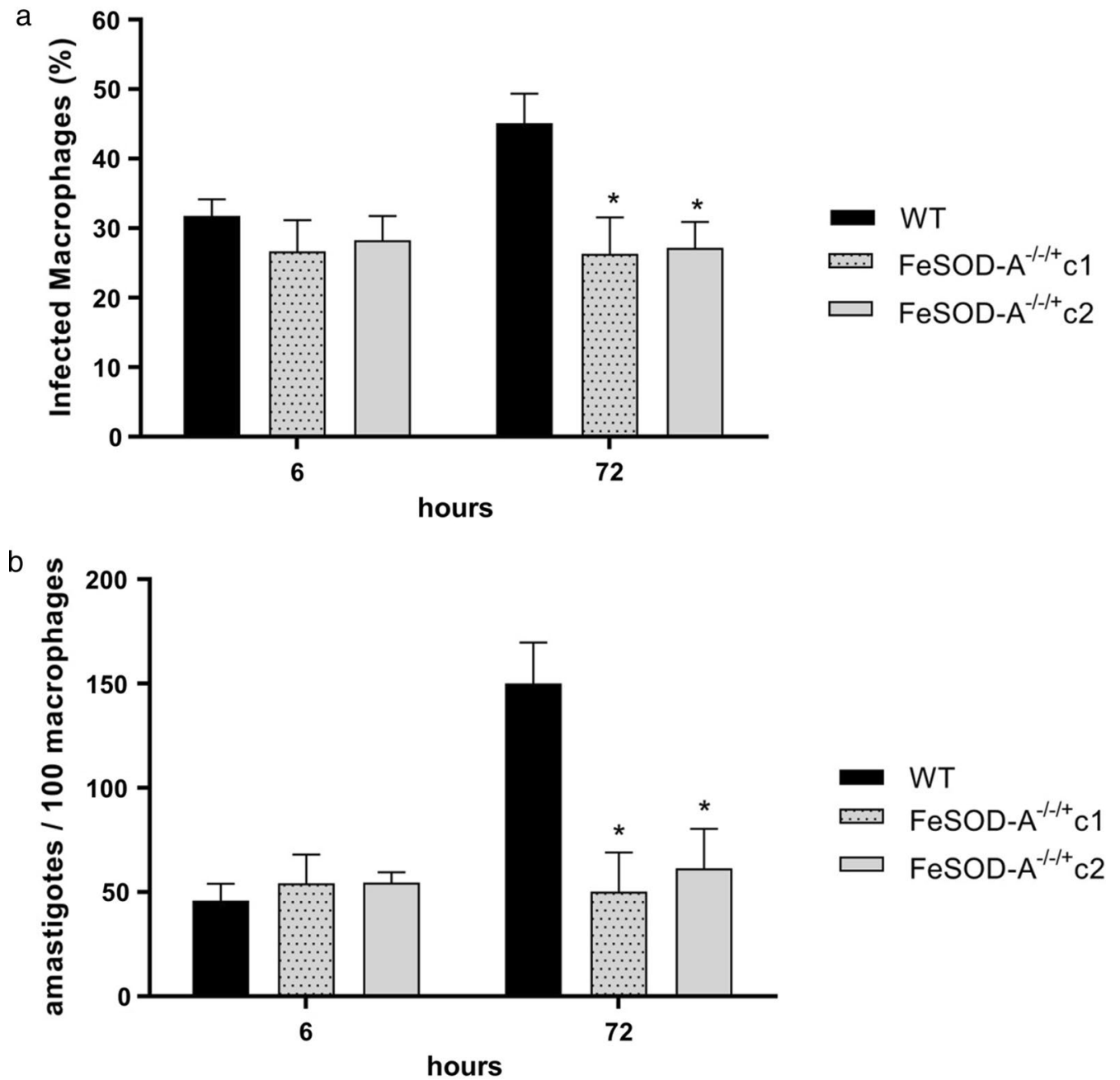

Fig. 4 Analysis of the infectivity of FeSOD- $A^{-/-/+}$mutants in THP-1 macrophages. Macrophages were infected with wild-type parasites and with clones FeSOD-A $A^{-/+}$in a ratio of 1:10 (10 parasites for each macrophage). Ordinary one-way ANOVA test with Bonferroni post hoc test was used to compare WT parasites and mutants at each time point; * represents significant differences in relation to the wild parasite $\left(^{*} p<0.05 ;{ }^{* *} p<0.01 ;{ }^{* * *}\right.$ $\left.p<0.001 ;{ }^{* * * *} p \leq 0.0001\right)$. a Percentage of infected macrophages, 6 and $72 \mathrm{~h}$ after infection. At $72 \mathrm{~h}-\mathrm{WT}$ vs. $C 1 F(2,8)=6.270 ; p=0.0351 ;$ at $72 \mathrm{~h}-$ WT vs. $C 2 F(2,8)=6.270 ; p=0.0306$. b Number of intracellular amastigotes per 100 macrophages, 6 and $72 \mathrm{~h}$ after infection. At $72 \mathrm{~h}-\mathrm{WT}$ vs. C1 F(2, 7) $=8.364 ; p=0.0164 ;$ at $72 h-W T$ vs. $C 2 F(2,7)=8.364 ; p=0.0286$

Here, we show that FeSOD-A $A^{-/ /+}$mutant L. infantum parasites were more susceptible to oxidative stress generated by menadione than the WT parasite. This result is consistent with previous studies that demonstrated the role of FeSOD-A in protection against oxidative stress in L. tropica and L. donovani [9], L. amazonensis [10], and L. infantum and L. braziliensis [12]. Furthermore, it was demonstrated that reduced expression of FeSOD-A in $L$. amazonensis resulted in mitochondrial oxidative damage and failure in promastigote-to-amastigote axenic differentiation [10]. Moreover, the lower infectivity of $\mathrm{FeSOD-}$ $A^{-/-/+}$parasites observed in our study is in agreement with the fact that they are less able to cope with oxidative stress from the host than the WT parasite. This result corroborates the previous finding that L. amazonensis with lower levels of FeSOD-A was unable to replicate in macrophages and did not generate lesions in mice [10]. The same study also showed that ROS is needed for parasite infectivity, and production of $\mathrm{H}_{2} \mathrm{O}_{2}$ by FeSOD-A is crucial in this process [10].

Despite the higher resistance of the mutants to $\mathrm{Sb}^{\mathrm{III}}$ and miltefosine, they were less tolerant to menadione, as expected. Menadione is included in the vitamin $\mathrm{K}$ class of compounds and generates oxidative stress by increasing peroxide and superoxide radical levels [27]. Therefore, this difference in phenotypes would be possibly owing to the different response of the mutants to drugs that act in multiple pathways, such as $\mathrm{Sb}^{\mathrm{III}}$ and 
miltefosine, as compared to their response to a compound that specifically generates oxidative stress such as menadione.

Several other enzymes not investigated in this study may also be involved in the FeSOD-A $A^{-/ /+}$mutant clone phenotypes, as different enzymes are known to be associated with the maintenance of redox states in different subcellular compartments [4]. We also emphasise that spontaneous dismutation of $\mathrm{O}_{2}^{-}$to $\mathrm{H}_{2} \mathrm{O}_{2}$ is rapid and that the catalysis of this reaction by SODs is important mainly to prevent $\mathrm{O}_{2}{ }^{-}$from reacting with cellular targets [28].

It is also very important to consider that here we only evaluated the transcript levels of some enzymes from the antioxidant defence system, but it is known that the transcript levels do not exactly match the protein expression in trypanosomatids. Leishmania genes are constitutively transcribed, and the regulation of gene expression largely occurs at the post-transcriptional level through RNA processing, RNA stability, translation efficiency, and post-translational modifications [29]. In addition, if FeSOD-A ${ }^{-/-/+}$mutant clones present other alterations in their genomes, such as small insertions or deletions (indels), the protein functions may be impaired or favoured. This may alter the phenotype of the parasite. Several interesting studies have demonstrated the association between specific mutations and drug resistance phenotypes. For example, mutations in the miltefosine transporter that imparts resistance to miltefosine and AMB [30-32], mutations in aquaglyceroporin that cause antimony resistance [33], and mutations in the calciumdependent protein kinase (CDPK1) are linked to paromomycin and antimony resistance [34]. Thus, we believe that it is possible that the FeSOD- $A^{-/-/+}$clones present changes that are undetectable through the analysis of only transcript levels.

\section{Conclusions}

Here, we demonstrate the importance of FeSOD-A in the maintenance of redox states in L. infantum. The downregulation of this enzyme caused the parasite to be more susceptible to oxidative stress and decreased its ability to maintain infection in macrophages. Furthermore, the decrease in FeSOD-A expression caused an imbalance in the pathways related to $\mathrm{Sb}^{\mathrm{III}}$ and miltefosine resistance.

\footnotetext{
Abbreviations

AMB: Amphotericin B; APX: Ascorbate peroxidase; FeSOD: Iron superoxide dismutase; GPX: Glutathione peroxidases; HYG: Hygromycin phosphotrans-

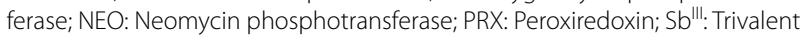
antimony; SbV: Pentavalent antimony; TXNPX: Tryparedoxin peroxidase.
}

\section{Supplementary Information}

The online version contains supplementary material available at https://doi. org/10.1186/s13071-021-04838-8.

\section{Additional file 1: Table S1. List of primers used in this study.}

Additional file 2: Figure S1. First attempt to knock out FeSOD-A using the CRISPR/Cas9 system. For this attempt, parasites bearing the pLPhygCas9 plasmid were transfected with the pSPneoHHsgRNAaH, containing sgRNA_144 or sgRNA_282, and with their respective donor DNA containing stop codons. Two other transfections were performed to provide more donor DNA for mutant parasites. The knockout was evaluated by PCR, using genomic DNA of wild-type parasites, of parasites expressing Cas 9 and of the mutant parasites (after one, two or three transfections with the donor DNA). The correct integration of the stop codons was evaluated by PCR by annealing a primer within the stop codon sequence and another primer within the FeSOD-A sequence. Two different guides were evaluated, a sgRNA144 and b sgRNA282. c After three transfections, the FeSOD-A protein levels were evaluated by western blot. MW: Molecular Weight Standard; bp, base pairs; NC: negative control; WT: wild-type; KDa, kilodalton

Additional file 3: Figure S2. Second attempt to knock out FeSOD-A using the CRISPR/Cas9 system. For this attempt, parasites bearing the pT007_ Cas9_T7 plasmid were transfected with DNA templates for the in vivo production of the sgRNAs and also with donor DNAs for allelic replacement of the FeSOD-A gene. Two different transfections were performed, one in which the parasites received only donor DNAs containing the NEO resistance marker and the other in which the parasites received donor DNAs containing both NEO and BDS markers. The donor DNAs are coloured in black. The correct integration of the resistance markers $\mathbf{a}$ NEO and $\mathbf{b}$ BSD was evaluated by PCR by annealing a primer in a 3'UTR region adjacent to the cassette (primer P1) and by another primer annealing within each resistance marker sequence (primers P2 or P6). The p1 primer is located 758 bp upstream of the FeSOD-A coding sequence. c Amplification of the FeSOD-A coding sequence by PCR using primers P4 and P5. d FeSOD-A protein levels were evaluated by western blot, comparing the wild-type and mutants. MW: Molecular Weight Standard; bp, base pairs; NC: negative control; WT: wild-type; KDa, kilodalton.

Additional file 4: Figure S3. Growth of WT, FeSOD-A $A^{-1+}$ and FeSOD$A^{-1-1+}$ parasites. Initially, $1 \times 10^{6}$ parasites per $\mathrm{mL}$ were inoculated in M199 medium. The parasites were cultivated and the growth was evaluated by daily counting of the parasites using the Z1 Coulter Counter. The data present the average of three independent experiments performed in triplicate, and the growth curves were built using a non-linear regression model with the "beta growth then decay" equation in GraphPad Prism v.8.2.0.

Additional file 5: Table S2. Probability to export FeSOD isoforms to mitochondria according to the results obtained at MitoProt $\|-v 1.101$.

Additional file 6: Table S3. Statistics of $\mathrm{IC}_{50}$ experiments. Two-way ANOVA dose vs. response-multiple comparisons.

\section{Acknowledgements}

The authors wish to thank Dr. Eva Gluenz (University of Glasgow, UK) for kindly providing the pTB007, pTNeo_v1 and pTBlast_v1 vectors; and Dr. Greg J Matlashewski (McGill University, Canada) for kindly providing the pSPneoHHsgR$\mathrm{NAaH}$ and pLPhygCas9 vectors. We thank also the Program for Technological Development in Tools for Health-PDTIS-FIOCRUZ for use of its facilities.

\section{Authors' contributions}

SMFM supervised the project. SMFM and AMMS conceived and designed the project. AMMS, PAS and IFMS carried out the experiments. SMFM, AMMS and PAS analysed the results. AMMS and SMFM wrote the manuscript. All authors read and approved the final manuscript.

\section{Funding}

This investigation received financial support from the following agencies: Programa INOVA FIOCRUZ-Fundação Oswaldo Cruz (VPPCB-007-FIO-18-2-94); 
Convênio Fiocruz-Institut Pasteur-USP (no grant number); Fundação de Amparo à Pesquisa do Estado de Minas Gerais (FAPEMIG_CBB-PPM and APQ02816-21), Convênio UGA/FAPEMIG (APQ-04382-16-D), Conselho Nacional de Desenvolvimento Científico e Tecnológico (CNPq 304158/2019-4), and Coordenação de Aperfeiçoamento de Pessoal de Nível Superior-Brasil (CAPES)_Finance Code 001. S.M.F. Murta is supported by CNPq. A.M.M. Santi and P.A. Silva are supported by CAPES and I.F.M. Santos by FAPEMIG.

\section{Availability of data and materials}

The datasets supporting the conclusions of this article are included within the article and its additional files.

\section{Declarations}

\section{Ethics approval and consent to participate}

Not applicable.

\section{Consent for publication}

Not applicable.

\section{Competing interests}

The authors declare that they have no competing interests.

Received: 30 March 2021 Accepted: 10 June 2021

Published online: 15 July 2021

\section{References}

1. WHO. Leishmaniasis. https://www.who.int/health-topics/leishmaniasis\# tab=tab 1. Accessed 16 June 2021.

2. Ponte-Sucre A, Gamarro F, Dujardin J, Barrett MP, Garcı R, Pountain AW, et al. Drug resistance and treatment failure in leishmaniasis: a 21st century challenge. PLoS Negl Trop Dis. 2017;11:e0006052.

3. Turrens JF. Oxidative stress and antioxidant defenses: a target for the treatment of diseases caused by parasitic protozoa. Mol Asp Med. 2004;25:211-20.

4. Castro H, Tomás AM. Peroxidases of trypanosomatids. Antioxid Redox Signal. 2008;10:1593-606.

5. Abreu IA, Cabelli DE. Superoxide dismutases - a review of the metal-associated mechanistic variations. Biochim Biophys Acta. 2010;1804:263-74.

6. Dufernez F, Yernaux C, Gerbod D, Noël C, Chauvenet M, Wintjens R, et al. The presence of four iron-containing superoxide dismutase isozymes in Trypanosomatidae: Characterization, subcellular localization, and phylogenetic origin in Trypanosoma brucei. Free Radic Biol Med. 2006;40:210-25.

7. Getachew F, Gedamu L. Leishmania donovani iron superoxide dismutase $\mathrm{A}$ is targeted to the mitochondria by its $\mathrm{N}$-terminal positively charged amino acids. Mol Biochem Parasitol. 2007;154:62-9.

8. Plewes KA, Barr SD, Gedamu L. Iron superoxide dismutases targeted to the glycosomes of Leishmania chagasi are important for survival. Infect Immun. 2003;71:5910-20.

9. Ghosh S, Goswami S, Adhya S. Role of superoxide dismutase in survival of Leishmania within the macrophage. Biochem J. 2003;369:447-52.

10. Mittra B, Laranjeira-silva MF, Miguel DC, Perrone J, de Menezes B, Andrews $\mathrm{N}$. The iron-dependent mitochondrial superoxide dismutase SODA promotes Leishmania virulence. J Biol Chem. 2017;292:12324-38.

11. Veronica J, Chandrasekaran S, Dayakar A, Devender M, Prajapati VK, Sundar S, et al. Iron superoxide dismutase contributes to miltefosine resistance in Leishmania donovani. FEBS J. 2019;286:3488-503.

12. Tessarollo NG, Andrade JM, Moreira DS, Murta SMF. Functional analysis of iron superoxide dismutase-A in wild-type and antimony-resistant Leishmania braziliensis and Leishmania infantum lines. Parasitol Int. 2015;64:125-9.

13. Liarte \& Murta. Selection and phenotype characterization of potassium antimony tartrate-resistant populations of four New World Leishmania species. Parasitol Res. 2010;107:205-12.
14. Robinson KA, Beverley SM. Improvements in transfection efficiency and tests of RNA interference (RNAi) approaches in the protozoan parasite Leishmania. Mol Biochem Parasitol. 2003;128:217-28.

15. Zhang W, Matlashewski G. CRISPR-Cas9-mediated genome editing in Leishmania donovani. MBio. 2015;6:e00861.

16. Beneke T, Madden R, Makin L, Valli J, Sunter J, Gluenz E. A CRISPR Cas9 high-throughput genome editing toolkit for kinetoplastids. R Soc Open Sci. 2017;4:170095.

17. Nogueira FB, Krieger MA, Nirdé P, Goldenberg S, Romanha AJ, Murta SMF. Increased expression of iron-containing superoxide dismutase-A (TcFeSOD-A) enzyme in Trypanosoma cruzi population with in vitro -induced resistance to benznidazole. Acta Trop. 2006;100:119-32.

18. Cruz AK, Titus R, Beverley SM. Plasticity in chromosome number and testing of essential genes in Leishmania by targeting. Proc Natl Acad Sci USA. 1993;90:1599-603.

19. Getachew F, Gedamu L. Leishmania donovani mitochondrial iron superoxide dismutase $A$ is released into the cytosol during miltefosine induced programmed cell death. Mol Biochem Parasitol. 2012;183:42-51.

20. Sterkers $Y$, Lachaud L, Bourgeois N, Crobu L, Bastien P, Pagès M. Novel insights into genome plasticity in Eukaryotes : Mosaic aneuploidy in Leishmania Micro Review Novel insights into genome plasticity in Eukaryotes : mosaic aneuploidy in Leishmania. Mol Microbiol. 2012;86:15-23.

21. Imamura $H$, Monsieurs $P$, Jara $M$, Sanders $M$, Maes I, Vanaerschot $M$ et al. Evaluation of whole genome amplification and bioinformatic methods for the characterization of Leishmania genomes at a single cell level. Sci Rep. 2020;10:15043.

22. Negreira GH, Monsieurs P, Imamura H, Maes I, Kuk N, Yagoubat A, et al. Exploring the evolution and adaptive role of mosaic aneuploidy in a clonal Leishmania donovani population using high throughput single cell genome sequencing. bioRxiv. 2020.03.05.976233.

23. Kumar A, Das S, Purkait B, Sardar AH, Ghosh AK, Dikhit MR, et al. Ascorbate peroxidase, a key molecule regulating amphotericin $B$ resistance in clinical isolates of Leishmania donovani. Antimicrob Agents Chemother. 2014;58:6172-84.

24. de Moreira SD, Xavier MV, Murta SMF. Ascorbate peroxidase overexpression protects Leishmania braziliensis against trivalent antimony effects. Mem Inst Oswaldo Cruz. 2018;113:e180377.

25. Ramos H, Valdivieso E, Gamargo M, Dagger F, Cohen BE. Amphotericin B kills unicellular Leishmanias by forming aqueous pores permeable to small cations and anions. J Membr Biol. 1996;152:65-75.

26. Purkait B, Kumar A, Nandi N, Sardar H, Das S, Kumar S, et al. Mechanism of amphotericin B resistance in clinical isolates of Leishmania donovani. Antimicrob Agents Chemother. 2012;56:1031-41.

27. Sakagami H, Satoh K, Hakeda YKM. Apoptosis-inducing activity of vitamin C and vitamin K. Cell Mol Biol. 2000;46:129-43.

28. Sheng Y, Abreu IA, Cabelli DE, Maroney MJ, Miller AF, Teixeira M, et al. Superoxide dismutases and superoxide reductases. Chem Rev. 2014;114:3854-918.

29. Grünebast J, Clos J. Leishmania: responding to environmental signals and challenges without regulated transcription. Comput Struct Biotechnol J.2020:18:4016-23.

30. Fernandez-Prada C, Vincent IM, Brotherton M, Rivas L, Leprohon P, Smith TK, et al. Different mutations in a P-type ATPase transporter in Leishmania parasites are associated with cross-resistance to two leading drugs by distinct mechanisms. PLoS Negl Trop Dis. 2016:10:e0005171.

31. Laffitte MCN, Leprohon P, Légaré D, Ouellette M. Deep-sequencing revealing mutation dynamics in the miltefosine transporter gene in Leishmania infantum selected for miltefosine resistance. Parasitol Res. 2016:115:3699-703.

32. Coelho AC, Boisvert S, Mukherjee A, Leprohon P, Corbeil J, Ouellette M. Multiple mutations in heterogeneous miltefosine- resistant Leishmania major population as determined by whole genome sequencing. PLoS Negl Trop Dis. 2012;6:e1512.

33. Potvin J-E, Leprohon P, Queffeulou M, Sundar S, Ouellette M. Mutations in an aquaglyceroporin as a proven marker of antimony clinical resistance in the parasite Leishmania donovani. Clin Infect Dis. 2020;22:ciaa1236. 
34. Bhattacharya A, Leprohon P, Bigot S, Padmanabhan PK, Mukherjee A, Roy $G$, et al. Coupling chemical mutagenesis to next generation sequencing for the identification of drug resistance mutations in Leishmania. Nat Commun. 2019;10:5627.

\section{Publisher's Note}

Springer Nature remains neutral with regard to jurisdictional claims in published maps and institutional affiliations.
Ready to submit your research? Choose BMC and benefit from:

- fast, convenient online submission

- thorough peer review by experienced researchers in your field

- rapid publication on acceptance

- support for research data, including large and complex data types

- gold Open Access which fosters wider collaboration and increased citations

- maximum visibility for your research: over $100 \mathrm{M}$ website views per year

At BMC, research is always in progress.

Learn more biomedcentral.com/submissions 